\title{
Neoadjuvant pembrolizumab with chemotherapy for the treatment of stage IIB-IIIB resectable lung squamous cell carcinoma
}

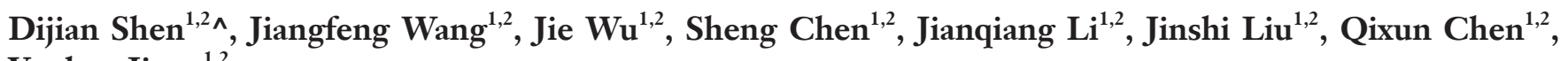 \\ Youhua Jiang ${ }^{1,2}$ \\ ${ }^{1}$ Department of Thoracic Surgery, Cancer Hospital of University of Chinese Academy of Sciences (Zhejiang Cancer Hospital), Hangzhou, China; \\ ${ }^{2}$ Institute of Cancer and Basic Medicine (IBMC), Chinese Academy of Science, Hangzhou, China \\ Contributions: (I) Conception and design: D Shen, Y Jiang, Q Chen; (II) Administrative support: Q Chen, Y Jiang, J Liu; (III) Provision of study \\ materials or patients: J Wu, J Wang, J Li, S Chen; (IV) Collection and assembly of data: D Shen, J Wang; (V) Data analysis and interpretation: D \\ Shen, J Wang, J Wu; (VI) Manuscript writing: All authors; (VII) Final approval of manuscript: All authors. \\ Correspondence to: Qixun Chen; Youhua Jiang. Department of Thoracic Surgery, Cancer Hospital of the University of the Chinese Academy of \\ Sciences (Zhejiang Cancer Hospital), No. 1 Banshan East Road, Gongshu District, Hangzhou 310022, China. \\ Email: chenqixun64@163.com; jiangyh@zjcc.org.cn.
}

\begin{abstract}
Background: Researches on programmed cell death (PD-1) as neoadjuvant immunotherapy for resectable non-small cell lung cancer is underway, which brings hope for individuals with the disease. However, a study dedicated to lung squamous cell carcinoma (LUSC) specifically has yet to be conducted. Now, data from our pilot prospective research neoadjuvant study provide new insights in the field of neoadjuvant regimen for LUSC.

Methods: Between June 2019 and July 2020, 37 adults with untreated, surgically resectable stage IIB-IIIB LUSC were enrolled into this prospective study. Patients received 2 cycles of pembrolizumab (2 mg/ $\mathrm{kg})$ with chemotherapy (albumin-bound paclitaxel $100 \mathrm{mg} / \mathrm{m}^{2}$ on days 1 and $8+$ carboplatin AUC 5) via intravenous administration every 3 weeks, and underwent surgical treatment 3-4 weeks after the second cycle. The primary endpoint of the study was the tumor pathologic complete response (pCR) rate. The toxicity profile, tumor major pathological remission, complete resection rate, response rate, and operative and postoperative complications were also evaluated.
\end{abstract}

Results: The postoperative pathological specimens of 17 (45.9\%) patients suggested pCR. Neoadjuvant pembrolizumab with chemotherapy had an acceptable side-effect profile, and no patients withdrew from the study preoperatively due to disease progression or toxicity. A major pathological response occurred in 24 (64.9\%) resected tumors. All tumors were completely resected (R0, 100\%). According to the Response Evaluation Criteria in Solid Tumors (RESIST), a response was evaluated before surgery in 32 (86.5\%) patients by computed tomography. Twenty-five (67.6\%) patients underwent thoracoscopic surgery. No deaths or postoperative major complications requiring reoperation occurred. Recurrence or metastasis was found in 2 patients during follow-up of 2-14 months.

Conclusions: The early outcomes of pembrolizumab with chemotherapy in the neoadjuvant setting as a novel treatment for resectable stage IIB-IIIB LUSC showed a high pCR rate that has not been seen previously, as well as a high R0 resection rate and a low toxicity profile. The long-term efficacy of this novel treatment and the validity of the present findings should be confirmed with longer follow-up and prospective comparative trials.

^ ORCID: 0000-0001-7132-7885. 
Keywords: Neoadjuvant chemoimmunotherapy; lung squamous cell carcinoma (LUSC); thoracic surgery; pembrolizumab

Submitted Nov 06, 2020. Accepted for publication Mar 04, 2021.

doi: $10.21037 /$ jtd-21-103

View this article at: http://dx.doi.org/10.21037/jtd-21-103

\section{Introduction}

Lung cancer is the most common malignancy worldwide. It has the largest impact of any cancer on morbidity and mortality among the world's population (1). Non-small cell lung cancer (NSCLC) make up $80-85 \%$ of newly diagnosed lung cancer cases (2). Despite the combination of multiple treatment modalities to treat resectable lung cancer, including chemotherapy, radiotherapy, and surgical resection, the prognosis of resectable NSCLC remains poor. For patients with stage III disease, the 5 -year survival rate is a meager $20 \%$ and recurrences are often observed after surgical treatment (3).

Immunotherapy has completely changed the approach to treating metastatic NSCLC. Particularly, antibodies that block programmed cell death 1 (PD-1) and programmed death-ligand 1 (PD-L1) have considerably improved the 5-year survival of NSCLC patients and show better tolerability than conventional chemotherapy (4-7). Pembrolizumab is a humanized monoclonal anti-PD-1 antibody that was approved in 2014 by the US Food and Drug Administration to treat melanoma. Since then, pembrolizumab has been approved to treat multiple malignancies. A large number of patients with metastatic NSCLC now receive pembrolizumab as a first-line therapy, either as monotherapy or as part of combined with chemotherapy. Furthermore, for patients with unresectable stage III NSCLC, PD-L1 sequential treatment following concurrent chemoradiotherapy has greatly improved progression-free survival and 3-year overall survival, bringing hope that the cure rates will also improve (8).

Since the mode of recurrence in patients with earlystage NSCLC who undergo complete resection is mainly systemic, there are high expectations for the integration of immunotherapy into the treatment approach. The first study to introduce a PD-1 inhibitor into a neoadjuvant regimen for resectable lung cancer was CheckMate 159. After 2 doses of nivolumab preoperatively, $45 \%$ of resected tumors (9/20) had a major pathological response, and $10 \%$ of patients $(2 / 20)$ even achieved a complete pathological response (pCR). Another phase II study used
Pembrolizumab is the NEOMUN trial which including 30 patients with NSCLC stage II/IIIA suitable for curative intent surgery (9). Furthermore, another clinical trial (NADIM study, NCT: 03081689) used 3 preoperative cycles of PD-1 inhibitor with chemotherapy to treat individuals with stage IIIA-N2 disease. This combination treatment proved to be majorly successful, with 35 patients (85.4\%) achieving major pathological response (MPR), of which 25 patients $(71.4 \%)$ were pCR. Moreover, among the 41 participants whose tumors were resected, 35 had not progressed at the 24-month follow-up (10). This suggests that immunotherapy combined with chemotherapy may be a better treatment model for resectable lung cancer and can achieve better results than traditional neoadjuvant chemotherapy.

However, different from the European and American populations, the epidermal growth factor receptor (EGFR) mutation rate of lung adenocarcinoma in Asian populations is as high as $40-60 \%$ (11), and a number of studies have shown that the effect of immunotherapy for patients with EGFR mutation is poor $(12,13)$.

In order to exclude the influence of high rates of EGFR mutations in lung adenocarcinoma cancer on the efficacy of neoadjuvant immunotherapy, this study was conducted to investigate the safety and treatment response of the pembrolizumab plus paclitaxel-carboplatin regimen for IIBIIIB LUSC. We present the following article in accordance with the STROBE reporting checklist (available at http:// dx.doi.org/10.21037/jtd-21-103).

\section{Methods}

\section{Study design}

A single-group study was designed and carried out at Cancer Hospital of University of Chinese Academy of Sciences (Zhejiang Cancer Hospital). All procedures performed in this study involving human participants were in accordance with the Declaration of Helsinki (as revised in 2013). The study was approved by institutional ethics committee of Zhejiang Cancer Hospital (No.: IRB-2021-16). The 
tumor stage was evaluated according to the American Joint Committee on Cancer $7^{\text {th }}$ edition criteria (3). Patients whose locally advanced tumors were evaluated as being surgically resectable by a multidisciplinary clinical team underwent treatment comprising 2 doses of pembrolizumab given intravenously at a dose of $2 \mathrm{mg}$ per kilogram of body weight every 3 weeks. Two cycles of chemotherapy were given simultaneously. The detailed regimen was as follows: albumin- paclitaxel $100 \mathrm{mg}$ per square meter of bodysurface area on days 1 and 8 ; and carboplatin targeted at an area under the curve of $5 \mathrm{mg}$ per milliliter per minute on day 1 . The operation was arranged to be performed 3-4 weeks after the second dose. Patients underwent chest enhanced computed tomography (CT) within the 1 week prior to the operation. Evaluation of tumor size-related changes was carried out using the Response Evaluation Criteria in Solid Tumors (RECIST), version 1.1 (14). The study's primary endpoint was the efficacy of anti-PD-1 therapy based on the evaluation of clinical and pathologic tumor response. The key secondary and exploratory endpoints were safety and feasibility. Subjects were kept under close monitoring for the occurrence of toxicity related to the neoadjuvant therapy, which was evaluated using the National Cancer Institute Common Terminology Criteria for Adverse Events version 5.0.

\section{Patients}

Patients were included on the basis of meeting the following criteria: (I) aged over 18 years old; and (II) with stage IIB to IIIB LUSC evaluated as being suitable for surgical resection prior to enrollment. All subjects were scored as 0 or 1 on the Eastern Cooperative Oncology Group (ECOG) performance status scale, with normal organ function and adequate pulmonary and cardiac function (15). Written informed consent was obtained from each patient. Patients were excluded on the basis of the following criteria: immunodeficiency disease; current systemic immunosuppressive therapy with either corticosteroids ( $>10 \mathrm{mg}$ daily prednisolone equivalent) or other immunosuppressive drugs; infectious disease; clinically significant concurrent malignancies; any previous medical treatment with immune checkpoint inhibitors (ICIs), or other antibodies or drugs targeting T-cell co-stimulation; a mental or psychological disorder that the investigator believed would affect the patient's ability to complete the study; acute or chronic hepatitis B or C virus infection; and hypersensitivity to albumin- paclitaxel and carboplatin.

\section{Surgery and follow-up}

Following neoadjuvant therapy, the patients underwent CT evaluation. Endoscopic evaluation is not necessary after neoadjuvant therapy. All eligible patients received radical surgery with curative intent. The selection of surgical procedures depended on individual patient factors (e.g., body weight, thoracic anatomy, and tumor location and size) and the surgeon's experience. The chest cavity was accessed through endoscopic surgery (video-assisted thoracoscopy, with 1 or 3 individually placed small utility incisions triangulated over the lateral chest wall) or through open surgery (with a horizontal incision made anterolaterally or posterolaterally over the $4^{\text {th }}$ or $5^{\text {th }}$ intercostal space). The patients in this research were scheduled to undergo at least anatomical lobectomy, including systemic and complete hilar, and mediastinal lymphadenectomy, as systemic lymph node dissection is an integral part of $\mathrm{R} 0$ resection. All operations were performed by 5 attending surgeons in our center, each of whom had experience of more than 1,000 cases of lung cancer surgery. Postoperative complications including hematological toxicity, bronchopleural fistula, pleural effusion, and lung infection were recorded on the case report form for up to 30 days after surgery. For the year immediately following surgery, subjects were examined every 3 months, and at 6-month intervals in the year after that.

\section{Statistical analyses}

Patients were kept under continuous monitoring for side effects, adverse events (AEs), and feasibility. If more than $25 \%$ of patients had a $90 \%$ or higher probability of delayed surgery, the treatment was considered unfeasible. The treatment was also deemed to be unsafe if the probability was $70 \%$ or more that the risk of grade 3 or 4 toxic effects was more than $25 \%$. Kaplan-Meier estimates were calculated for progression-free survival and overall survival along with $95 \%$ confidence intervals (CIs). The median follow-up time was obtained by adopting the reverse Kaplan-Meier method. Categorical variables were expressed as absolute frequency and relative frequency, and numerical variables were expressed as means (SD) or medians. Recurrence-free survival-defined as the time from the date of the operation until the occurrence of recurrent disease or death-was calculated using the Kaplan-Meier method. $\mathrm{P}$ values were bilateral, and all analysis was performed with 0.05 as the threshold for significance unless indicated otherwise. 
Table 1 Characteristics of the patients at baseline

\begin{tabular}{|c|c|}
\hline Characteristics & Value \\
\hline \multicolumn{2}{|l|}{ Age (yr) } \\
\hline Median & 62.8 \\
\hline Range & $38-76$ \\
\hline \multicolumn{2}{|l|}{ Sex, n (\%) } \\
\hline Male & 35 (94.6) \\
\hline Female & $2(5.4)$ \\
\hline \multicolumn{2}{|c|}{ Smoking status, n (\%) } \\
\hline Never & $6(16.2)$ \\
\hline Former or current & $31(83.8)$ \\
\hline \multicolumn{2}{|c|}{$\begin{array}{l}\text { Tumour, Node, Metastasis staging } \\
\text { classification, } \mathrm{n}(\%)\end{array}$} \\
\hline T1N2M0 & $1(2.7)$ \\
\hline T2N1M0 & $2(5.4)$ \\
\hline T2N2M0 & $9(24.3)$ \\
\hline T3NOMO & $1(2.7)$ \\
\hline T3N1M0 & $14(37.8)$ \\
\hline T3N2MO & $6(16.2)$ \\
\hline T4NOMO & $1(2.7)$ \\
\hline T4N1M0 & $3(8.1)$ \\
\hline
\end{tabular}

\section{Results}

\section{Patient characteristics}

Between June 1, 2019 and July 30, 2020, 37 patients were enrolled into the study, all of whom completed 2 cycles of neoadjuvant therapy. Complete tumor resection was obtained by every patient, and no related cases of morbidity or mortality were recorded. Baseline characteristics of the patients who met the study's eligibility criteria are described in Table 1. These patients had a median age of 62.8 years, and included $3(8.1 \%)$ stage IIB, 28 (75.7\%) stage IIIA, and $6(16.2 \%)$ stage IIIB cases. Thirty-one $(83.8 \%)$ patients were current or former smokers.

\section{Safety and feasibility of neoadjuvant therapy}

All patients completed 2 cycles of pembrolizumab therapy. In 4 patients, the dose of chemotherapy drugs was reduced due to bone marrow suppression. None of the patients quit the course of neoadjuvant therapy due to toxic effects or disease progression. No previously unreported toxicities were observed in relation to the combined neoadjuvant treatment. AEs relating to the treatment were experienced by $70.3 \%(26 / 37)$ of subjects (Table 2). In terms of grade 3 AEs, there were $2(5.4 \%)$ cases of neutropenia, 1 (2.7\%) case of thrombocytopenia, and $1(2.7 \%)$ case of increased aminotransferases. No grade $\geq 4$ AEs were observed. CT was performed on all patients before surgery to evaluate the treatment effect. Complete and partial responses were observed in $10(27.0 \%)$ and $22(59.5 \%)$ patients, respectively. There were $5(13.5 \%)$ patients whose disease was stable, and no cases of disease progression were recorded.

\section{Surgery}

All patients achieved complete resection (R0). Twenty-five $(67.6 \%)$ patients underwent video-assisted thoracoscopy surgery, and $12(32.4 \%)$ received open surgery. The specific surgical procedures performed were as follows: lobectomy (22 cases, 59.5\%); combined lobectomy [7 cases, $18.9 \%$; including right middle and lower lobectomy (5 cases, $13.5 \%$ ) and right upper and middle lobectomy (2 cases, 5.4\%)]; pulmonary sleeve resection [6 cases, $16.2 \%$; including $1(2.7 \%)$ combined with pulmonary artery reconstruction], and left pneumonectomy (2 cases, $5.4 \%$ ). The mean operative time was 184 minutes (range, 100-310 minutes).

Postoperative complications are summarized in Table 3. None of the participants experienced severe intraoperative complications or died in hospital. No major postoperative complications requiring reoperation occurred. During the postoperative period, the most common complications were pneumothorax (9 cases, $24.3 \%$ ) and pleural effusion ( 3 cases, $8.1 \%$ ). At a median of 7 months (range, 2-14 months) postoperative follow-up. Of 37 participants, 35 (94.6\%) who had received R0 resection remained alive and were without recurrence. Bone metastasis was detected in 1 subject at 4 months after surgery, while another was found to have brain metastases at 9 months after surgery.

\section{Pathological assessment}

From the 37 patients who underwent surgery, a median of 21 (range, 11-37) lymph nodes were resected. Resected specimens from 17 (45.9\%) patients displayed pathological complete response (ypT0N0; with y indicating neoadjuvant therapy and $\mathrm{p}$ indicating pathological assessment). A major 
Table 2 Adverse events during neoadjuvant chemoimmunotherapy

\begin{tabular}{|c|c|c|}
\hline Events of during neoadjuvant therapy, $\mathrm{n}(\%)$ & Grade 1-2 & Grade 3 \\
\hline Neutropenia & $6(16.2)$ & $2(5.4)$ \\
\hline Decreased appetite & $6(16.2)$ & - \\
\hline Arthralgia & $6(16.2)$ & - \\
\hline Constipation & $5(13.5)$ & - \\
\hline Nausea & $5(13.5)$ & - \\
\hline Anemia & $4(10.8)$ & - \\
\hline Diarrhoea & $4(10.8)$ & - \\
\hline Increased aminotransferases & $3(8.1)$ & $1(2.7)$ \\
\hline Thrombocytopenia & $2(5.4)$ & $1(2.7)$ \\
\hline Hypothyroidism & $1(2.7)$ & - \\
\hline
\end{tabular}

Table 3 Postoperative complications

\begin{tabular}{lc}
\hline Postoperative events & $\mathrm{n}(\%)$ \\
\hline Pneumothorax & $9(24.3)$ \\
Pleural effusion & $3(8.1)$ \\
Postoperative bleeding & $2(5.4)$ \\
Pneumonia & $2(5.4)$ \\
Atrial fibrillation & $2(5.4)$ \\
Recurrent nerve paralysis & $2(5.4)$ \\
Chylothorax & $1(2.7)$ \\
Hypokalemia & $1(2.7)$ \\
Increased aminotransferases & $1(2.7)$ \\
\hline
\end{tabular}

pathological response was found in 24 (64.9\%) resected tumors. The pathological tumor regression in all resection specimens compared with preoperative CT evaluation are listed in Figure 1. Preoperative CT did not fully reflect the final pathological remission status among the patients in our study.

\section{Discussion}

In this single-center, prospective trial, we administrated the neoadjuvant treatment regimen of pembrolizumab combined with chemotherapy to patients with potentially curable locally advanced LUSC. Two cycles of this regimen, which was given via intravenous injection, resulted in few immediate AEs and no severe AEs. Furthermore, no scheduled procedures were delayed, and $45.9 \%$ of tumors achieved pCR. No serious immune-related AEs (irAEs), such as myocarditis or hepatitis, were observed in our patients, which may be attributable to their good physical conditions compared with advanced or metastatic patients. The pCR rate of pembrolizumab combined with chemotherapy was far higher than that of traditional neoadjuvant chemotherapy $(16,17)$ or neoadjuvant immunotherapy alone. A meta-analysis showed that neoadjuvant immunotherapy had a pCR rate of $11.76 \%$ (18). This result implies that neoadjuvant chemoimmunotherapy might achieve a better tumor response and be more promising as a treatment regimen than neoadjuvant immunotherapy alone.

Unlike other studies, we only selected patients with LUSC. As we know, Asian patients with lung adenocarcinomas show a much higher frequency of EGFR active mutations, with the overall EGFR mutation frequency in mainland Chinese population reaching $50.2 \%$ (11). A number of clinical studies $(12,13,19,20)$ have shown that immunotherapy for EGFR mutations in NSCLC has poor efficacy; therefore, in this study, we 

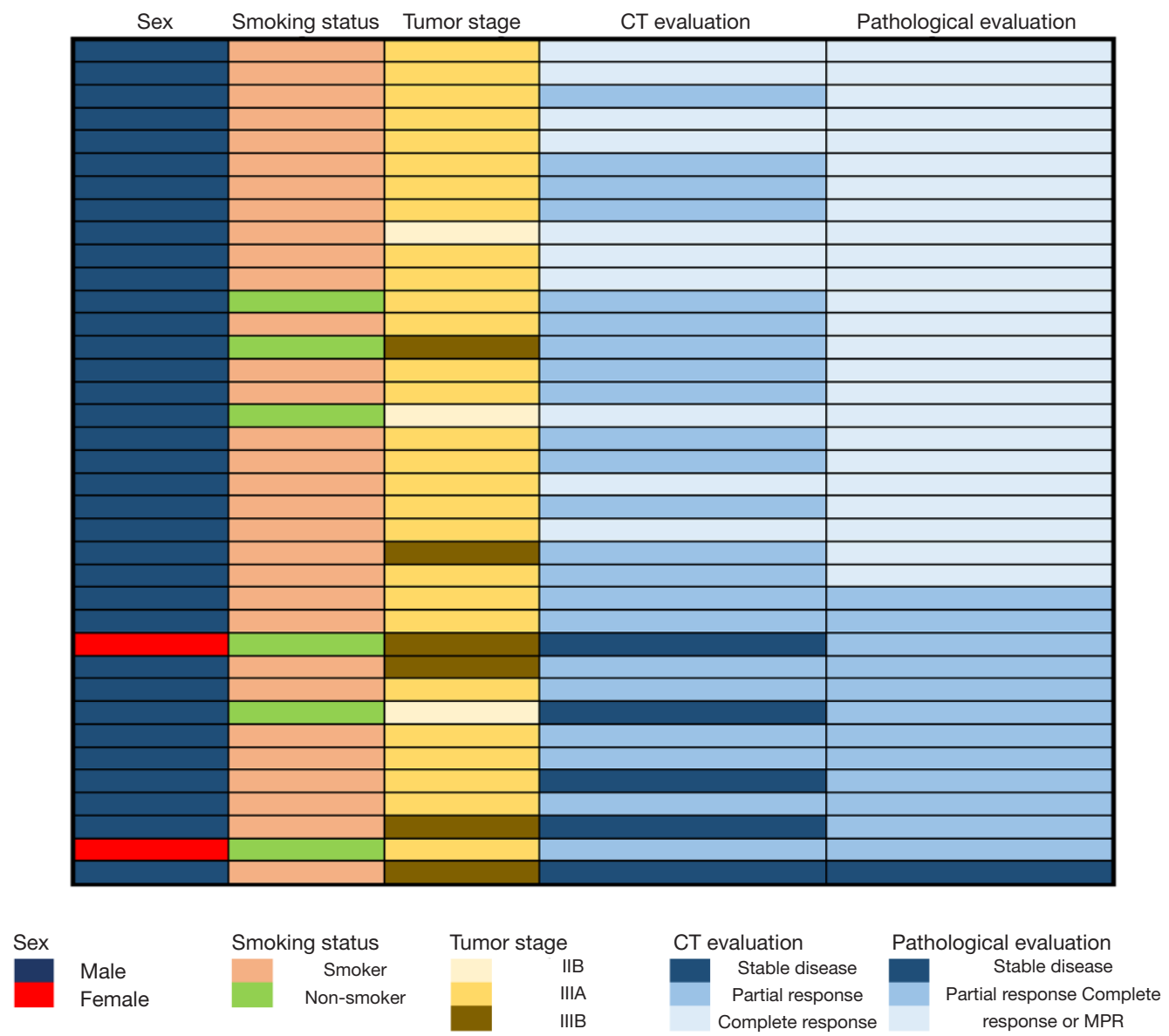

Figure 1 Patients' characteristics and preoperative CT evaluation compared with the final pathological evaluation.

temporarily excluded patients with lung adenocarcinoma. The efficacy of neoadjuvant chemoimmunotherapy for patients with EGFR-mutant lung adenocarcinoma requires more specific research.

In the NADIM study, the rates of MPR and pCR reached $76.1 \%$ and $54.3 \%$ for stage IIIA patients in all study groups (10), which were similar to those observed in our research. However, patients in the NADIM study received 3 cycles of the regimen before surgical resection; consequently, they showed a higher incidence $(93.5 \%)$ of neoadjuvant therapy-related AEs, with $30.4 \%$ of patients (14/46) suffering AEs of at least grade 3 (21). Therefore, we propose believe that extended neoadjuvant therapy cycles are associated with an increased incidence of treatment-related AEs. Further clinical randomized controlled trials are needed to clarify the benefits and risks associated with the number of cycles of neoadjuvant chemoimmunotherapy used.

For surgeons, whether neoadjuvant chemoimmunotherapy affects the difficulty of surgery is a matter of concern.
In our study, $67.6 \%$ patients underwent video-assisted thoracoscopy surgery. None of them had severe intraoperative complications, and the mean operative time was 184 minutes. The data showed us that neoadjuvant chemoimmunotherapy did not significantly increase the difficulty of the surgical procedure. Based on our experience, 3-4 weeks after the second dose of chemoimmunotherapy is a good time to perform surgery; at this point, we did not find any severe adhesions or hilar fibrosis during the procedures.

Before neoadjuvant chemoimmunotherapy can become the standard treatment strategy for locally advanced lung cancer, there are still some challenges to overcome. Firstly, effective biomarkers to predict the efficacy of neoadjuvant therapy are lacking. PD-L1 level is an established biomarker for the efficacy prediction of PD-1 checkpoint inhibitors in NSCLC $(22,23)$; however, the relationship of PD-L1 level with pathological reaction in the neoadjuvant setting is still surrounded by controversy. The NeoStar 
study indicated PD-L1 and pathological reaction to be correlated; compared with non-responders, responders had an increased median PD-L1 level prior to therapy ( $80 \%$ vs. 1\%) (24). However, other researchers reported no such correlation (25-27). For instance, in the NADIM clinical trial, $58 \%$ of patients whose PD-L1 tumor proportion score was below $25 \%$ had a MPR or pCR. Another biomarker, tumor mutational burden (TMB), also has the problem of drawing different conclusions in different studies. Forde et al. confirmed patients' pathological responses to be significantly correlated with their pretherapy TMB (25), whereas another study arrived at a different conclusion (28). Thus, other effective biomarkers for this treatment strategy urgently need to be identified. The strategy of adjuvant treatment is another unresolved problem. The PACIFIC trial evaluated the activity and safety through a comparison of consolidation therapy with durvalumab versus a placebo in patients with stage III, locally advanced, unresectable NSCLC who were without progression following standard chemoradiotherapy. The result was encouraging, with durvalumab significantly outperforming the placebo in terms of progression-free survival (8). When it comes to the adjuvant treatment, there is still no strong evidence of ICIs adjuvant therapy. Adjuvant treatment regimens are still controversial regarding whether chemotherapy can be used in combination or whether only PD-1/PDL-1 blockade should be used. Furthermore, the optimal maintenance time for adjuvant therapy has yet to be clarified. For this reason, we did not stipulate the regimen of adjuvant therapy in our study.

Some retrospective analyses have shown a relationship between the occurrence of MPR after neoadjuvant chemotherapy and recurrence-free and improved overall survival $(29,30)$. In this context, the MPR rate $(67.6 \%)$ observed among our patients is promising. During a median postoperative follow-up of 7 months, none of the patients who achieved MPR experienced recurrence. However, long-term follow-up is needed to determine progressionfree survival and overall survival with this treatment.

Our study has a number of limitations; these include but are not limited to the short postoperative follow-up time and the small number of patients enrolled and we did not monitor the patient's immune status during treatment. Neoadjuvant chemoimmunotherapy holds promise as a treatment for resectable stage IIB-IIIB LUSC, but we still need to pay close attention to the immune related AEs during therapy. Larger studies and longer follow-up, as well as more prospective comparative trials, are needed to ascertain the optimal duration of neoadjuvant treatment and to identify the best biomarkers for predicting therapeutic efficacy.

\section{Conclusions}

This study shows that as a new treatment regimen for resectable stage IIB-IIIB LUSC, the early outcome of pembrolizumab with chemotherapy in a neoadjuvant setting showed an unprecedented high PCR rate, high $\mathrm{R} 0$ resection rate and low toxicity. The long-term efficacy of this new therapy and the effectiveness of the current findings should be confirmed through longer follow-up and prospective comparative trials.

\section{Acknowledgments}

Funding: None.

\section{Footnote}

Reporting Checklist: The authors have completed the STROBE reporting checklist. Available at http://dx.doi. org/10.21037/jtd-21-103

Data Sharing Statement: Available at http://dx.doi. org/10.21037/jtd-21-103

Conflicts of Interest: All authors have completed the ICMJE uniform disclosure form (available at http://dx.doi. org/10.21037/jtd-21-103). The authors have no conflicts of interest to declare.

Ethical Statement: The authors are accountable for all aspects of the work in ensuring that questions related to the accuracy or integrity of any part of the work are appropriately investigated and resolved. All procedures performed in this study involving human participants were in accordance with the Declaration of Helsinki (as revised in 2013). The study was approved by institutional ethics committee of Zhejiang Cancer Hospital (No.: IRB-2021-16). Written informed consent was obtained from each patient.

Open Access Statement: This is an Open Access article distributed in accordance with the Creative Commons Attribution-NonCommercial-NoDerivs 4.0 International License (CC BY-NC-ND 4.0), which permits the noncommercial replication and distribution of the article with the strict proviso that no changes or edits are made and the original work is properly cited (including links to both the 
formal publication through the relevant DOI and the license). See: https://creativecommons.org/licenses/by-nc-nd/4.0/.

\section{References}

1. Bray F, Ferlay J, Soerjomataram I, et al. Global cancer statistics 2018: GLOBOCAN estimates of incidence and mortality worldwide for 36 cancers in 185 countries. CA Cancer J Clin 2018;68:394-424.

2. Yeh J, Marrone KA, Forde PM. Neoadjuvant and consolidation immuno-oncology therapy in stage III non-small cell lung cancer. J Thorac Dis 2018;10:S451-S459.

3. Goldstraw P, Crowley J, Chansky K, et al. The IASLC Lung Cancer Staging Project: proposals for the revision of the TNM stage groupings in the forthcoming (seventh) edition of the TNM Classification of malignant tumours. J Thorac Oncol 2007;2:706-14.

4. Gettinger SN, Horn L, Gandhi L, et al. Overall Survival and Long-Term Safety of Nivolumab (Anti-Programmed Death 1 Antibody, BMS-936558, ONO-4538) in Patients With Previously Treated Advanced Non-Small-Cell Lung Cancer. J Clin Oncol 2015;33:2004-12.

5. Rittmeyer A, Barlesi F, Waterkamp D, et al. Atezolizumab versus docetaxel in patients with previously treated non-small-cell lung cancer (OAK): a phase 3, openlabel, multicentre randomised controlled trial. Lancet 2017;389:255-65.

6. Reck M, Rodrdrarlesi F, Waterkamp D, et al. Pembrolizumab versus Chemotherapy for PD-L1Positive Non-Small-Cell Lung Cancer. N Engl J Med 2016;375:1823-33.

7. Garon EB, Rizvi NA, Hui R, et al. Pembrolizumab for the treatment of non-small-cell lung cancer. N Engl J Med 2015;372:2018-28.

8. Antonia SJ, Villegas A, Daniel D, et al. Durvalumab after chemoradiotherapy in stage III non-small-cell lung cancer. N Engl J Med 2017;377:1919-29.

9. Eichhorn F, Klotz LV, Bischoff H, et al. Neoadjuvant antiprogrammed Death-1 immunotherapy by Pembrolizumab in resectable nodal positive stage II/IIIa non-small-cell lung cancer (NSCLC): the NEOMUN trial. BMC Cancer 2019;19:413.

10. Provencio $M$, Nadal E, Insa A, et al. OA13.05 NADIM Study: Updated Clinical Research and Outcomes. J Thorac Oncol 2019;14:S241.

11. Shi Y, Li J, Zhang S, et al. Molecular Epidemiology of EGFR Mutations in Asian Patients with Advanced Non-
Small-Cell Lung Cancer of Adenocarcinoma Histology Mainland China Subset Analysis of the PIONEER study. PLoS One 2015;10:e0143515.

12. Herbst RS, Baas P, Kim DW, et al. Pembrolizumab versus docetaxel for previously treated, PD-L1-positive, advanced non-small-cell lung cancer (KEYNOTE-010): a randomised controlled trial. Lancet 2016;387:1540-50.

13. Lisberg A, Cummings A, Goldman JW, et al. A Phase II Study of Pembrolizumab in EGFR-Mutant, PDL1+, Tyrosine Kinase Inhibitor $\mathrm{Na} \mathrm{Nal}$ lung cancer (KEYNOTE-010): a randomised controlled trial. J Thorac Oncol 2018;13:1138-45.

14. Eisenhauer EA, Therasse P, Bogaerts J, et al. New response evaluation criteria in solid tumours: revised RECIST guideline (version 1.1). Eur J Cancer 2009;45:228-47.

15. Oken MM, Creech RH, Tormey DC, et al. Toxicity and response criteria of the Eastern Cooperative Oncology Group. Am J Clin Oncol 1982;5:649-55.

16. Detterbeck FC, Socinski MA, Gralla RJ, et al. Neoadjuvant chemotherapy with gemcitabine-containing regimens in patients with early-stage non-small cell lung cancer. J Thorac Oncol 2008;3:37-45.

17. Xu YP, Li B, Xu XL, et al. Is There a Survival Benefit in Patients With Stage IIIA (N2) Non-small Cell Lung Cancer Receiving Neoadjuvant Chemotherapy and/or Radiotherapy Prior to Surgical Resection: A Systematic Review and Meta-analysis. Medicine (Baltimore) 2015;94:e879.

18. Jia XH, Xu H, Geng LY, et al. Efficacy and safety of neoadjuvant immunotherapy in resectable nonsmall cell lung cancer: A meta-analysis. Lung Cancer 2020;147:143-53.

19. Borghaei H, Paz-Ares L, Horn L, et al. Nivolumab versus Docetaxel in Advanced Nonsquamous Non-Small-Cell Lung Cancer. N Engl J Med 2015;373:1627-39.

20. Oxnard GR, Yang JC, Yu H, et al. TATTON: a multi-arm, phase Ib trial of osimertinib combined with selumetinib, savolitinib, or durvalumab in EGFR-mutant lung cancer. Ann Oncol 2020;31:507-16.

21. Provencio M, Nadal E, Insa A, et al. Neoadjuvant chemotherapy and nivolumab in resectable non-small-cell lung cancer (NADIM): an open-label, multicentre, singlearm, phase 2 trial. Lancet Oncol 2020;21:1413-22.

22. Bradley CA. Pembrolizumab improves OS across PD-L1 subgroups. Nat Rev Clin Oncol 2019;16:403.

23. Miller PG, Li G, Singal G. PD-L1 Status and Survival in Patients With Lung Cancer-Reply. JAMA 2019;322:783-4.

24. Cascone T, William WN, Weissferdt A, et al. Neoadjuvant nivolumab (N) or nivolumab plus ipilimumab (NI) for 
resectable non-small cell lung cancer (NSCLC): Clinical and correlative results from the NEOSTAR study. J Clin Oncol 2019;37:abstr 8504.

25. Forde PM, Chaft JE, Smith KN, et al. Neoadjuvant PD-1 Blockade in Resectable Lung Cancer. N Engl J Med 2018;378:1976-86.

26. Bar J, Urban D, Ofek E, et al. Neoadjuvant pembrolizumab (Pembro) for early stage non-small cell lung cancer (NSCLC): Updated report of a phase I study, MK3475-223. J Clin Oncol 2019;37:abstr 8534.

27. Shu CA, Gainor JF, Awad MM, et al. Neoadjuvant atezolizumab and chemotherapy in patients with resectable non-small-cell lung cancer: an open-label, multicentre, single-arm, phase 2 trial. Lancet Oncol 2020;21:786-95.

Cite this article as: Shen D, Wang J, Wu J, Chen S, Li J, Liu J, Chen Q, Jiang Y. Neoadjuvant pembrolizumab with chemotherapy for the treatment of stage IIB-IIIB resectable lung squamous cell carcinoma. J Thorac Dis 2021;13(3):1760-1768. doi: $10.21037 /$ jtd-21-103
28. Garassino M, Rodriguez-Abreu D, Gadgeel S, et al. OA04.06 Evaluation of TMB in KEYNOTE-189: Pembrolizumab Plus Chemotherapy vs Placebo Plus Chemotherapy for Nonsquamous NSCLC. J Thorac Oncol 2019;14:S216-S217.

29. Pataer A, Kalhor N, Correa AM, et al. Histopathologic response criteria predict survival of patients with resected lung cancer after neoadjuvant chemotherapy. J Thorac Oncol 2012;7:825-32.

30. Hellmann MD, Chaft JE, William WN Jr, et al. Pathological response after neoadjuvant chemotherapy in resectable non-small-cell lung cancers: proposal for the use of major pathological response as a surrogate endpoint. Lancet Oncol 2014;15:e42-50. 